\title{
Medical Students' Perspectives of Remote Residency Interviews
}

\author{
Tanha Shah $^{1}$ (D) $\cdot$ Kennedy Werner ${ }^{1} \cdot$ Mary Morreale $^{1} \cdot$ Cynthia Arfken $^{1}$
}

Received: 23 August 2021 / Accepted: 26 January 2022 / Published online: 19 February 2022

(C) Academic Psychiatry 2022

To the Editor:

Due to the COVID-19 pandemic, psychiatry residency interviews were held remotely in 2020. A recent commentary [1, 2] highlighted advantages and disadvantages of such virtual interviews and advocated for continued use. Unfortunately, there is sparse research on students who participated in remote residency interviewing [3]. Understanding their experiences may highlight areas of improvement for future virtual interviews.

To assess students' experiences with the virtual interview process, two trained $3^{\text {rd }}$ year medical students conducted 30 minute interviews with eight of the sixteen $4^{\text {th }}$ year medical students from Wayne State University who matched into a psychiatry program via the National Resident Matching Program. The age of participants ranged from 25 to 36, half were female, and six were Asian or Middle Eastern and two were Caucasian. Interviews were conducted, audio-recorded, and transcribed on a video conferencing platform. Transcripts were reviewed for accuracy by both the trained interviewers and participants. The interview guide followed a temporal sequence of interview to promote recall with pre-specified prompts (e.g., videoconferencing fatigue). Thematic analysis was used with the team members reviewing all transcripts. The Wayne State University Institutional Review Board approved the study with one participant selected to win a $\$ 100$ gift certificate.

All participants mentioned positive and negative aspects of remote interviewing. Positive aspects of the process included savings of time and money as participants did not need to make travel arrangements. Participants valued the costsavings associated with remote interviews, with one stating, "I absolutely would have had to take out extra loans if traveling was required for interviews." They appreciated the opportunity to schedule interviews back-to-back without having to

Tanha Shah

ec8999@wayne.edu

1 Wayne State University School of Medicine, Detroit, MI, USA factor in additional travel time [4]. Some participants valued the pre-interview activity, noting that it added a caring touch to the interview experience, which might positively impact their rank list decision. One student appreciated when these activities were done with a small number of individuals present, stating, "And then they split us into groups of two of us with two residents, so it was a really small group so you could actually ask stuff and have a conversation...."

Negative aspects of remote interviews included the inability to physically view the hospital or city in which programs were located and meeting current residents. One participant said, "I just want to see what their culture is like. What are they into? Do they get along? What do they hate?". Some participants mentioned dissatisfaction with virtual pre-interview socials event. One reported, "I've been looking forward to these dinners ... you just get to mingle, talk, network with all the residents and other applicants and I was so excited for that. And there's just not really a way to do that on [videoconference platform]." Another participant noted that game-type activities meant to "break the ice" were awkward on a virtual platform. For the interviews themselves, participants mentioned the drawback of using multiple platforms, receiving schedules and links last minute, and requiring the participant to enter a breakout room as opposed to the program sending them there. Although no participants spontaneously reported fatigue related to remote interviews, several mentioned this when prompted. One student noted it "just felt like being on stage for too long."

In response to prompts, participants noted Zoom as the easiest platform to navigate due to extensive classroom use at this institution. Participants did highlight the additional difficulty of knowing when it was time to unmute and the discomfort of viewing oneself on display, especially when they were on the conference with many others. Some participants mentioned moving to a different location for their interview (e.g., parent's house) to use faster and more stable internet connection; some participants bought specialized lighting. Participants mentioned knowing students who used space and technology provided by the university which is beneficial to students with limited resources and financial inequities. As 
for the backdrop, opinions varied with some participants creating a neutral background and others wanting to emphasize the uniqueness of their interview space, which "stirred up a lot of good, really organic conversations that lead into talking about hobbies." One participant admitted to wearing a professional top and pajama bottoms.

When prompted, participants suggested that they would have done better on the interview in person because social cues and body language are not easily read online. One participant said, "the ability to connect with your interviewer kind of sometimes gets lost through the technology." Some participants stated that they were afraid to ask personal questions during the interview, because of a possible breach in privacy (recorded meetings or program directors being present in the background).

Limitations include the small sample size at one institution and only interviewing students who successfully matched into psychiatry. As a qualitative study, the intent was to discover students' perspectives and detect themes, not to generalize findings as in a quantitative survey with a well-defined population. This study took place near the end of the academic year while students were in the process of relocating, which made some eligible students difficult to contact.

Overall, participants seemed to put extensive thought and effort into their virtual interview preparation and considered technology, backdrops, dress, lighting, and internet connection to eliminate potential issues or disturbances. Given identified concerns, program directors might consider adding virtual tours of facilities, so students can get a sense of the environment in which they will be working, as well video blogs from current residents to reveal the workplace culture [5]. To make the pre-interview events more conducive to intimate conversation, a smaller number of students might be placed in breakout rooms with 1-2 residents. To ease students' anxiety, programs might send out a concise schedule of the interview day with appropriate links well in advance and confirm that the interview is not being recorded or observed. Given complaints of fatigue associated with the remote interview process, a reduced number of interviews and scheduled breaks should be considered. Finally, for those students who are unable to secure an appropriate setting and lack needed technology, medical school administration should provide these.

Funding This study received funding from the Department of Psychiatry, Wayne State University School of Medicine.

Declarations This study was expedited and exempt by the IRB at Wayne State University.

Disclosures On behalf of all authors, the corresponding author states that there is no conflict of interest.

\section{References}

1. Hammoud MM, Standiford TC, Carmody JB. The 2020-2021 residency application cycle: lessons learned and lingering problems: Lessons learned and lingering problems. JAMA. 2021;325(22): 2249-50.

2. Grova MM, Donohue SJ, Meyers MO, Kim HJ, Ollila DW. Direct comparison of in-person versus virtual interviews for complex general surgical oncology fellowship in the COVID-19 era. Ann Surg Oncol. 2021;28(4):1908-15.

3. Patel TY, Bedi HS, Deitte LA, Lewis PJ, Marx MV, Jordan SG. Brave new world: challenges and opportunities in the COVID-19 virtual interview season. Acad Radiol. 2020;27(10):1456-60.

4. Rekawek P, Graves LL, Panchal N, Schlieve T. Navigating the virtual match: pitfalls of the virtual interview. J Oral Maxillofac Surg: J Oral Maxillofac Surg. 2021;79(3):516-7.

5. Zertuche JP, Connors J, Scheinman A, Kothari N, Wong K. Using virtual reality as a replacement for hospital tours during residency interviews. Med Educ Online. 2020;25(1):1777066.

Publisher's Note Springer Nature remains neutral with regard to jurisdictional claims in published maps and institutional affiliations. 